\title{
Comparative Analysis of Safety and Effect of Minimally Invasive Esophageal Cancer Radical Resection and Conventional Thoracotomy for Esophageal Cancer
}

\author{
$\mathrm{Ke} \mathrm{Du}^{*}$, Zhenxing Wang \\ Department of Thoracic Surgery, Liaocheng People's Hospital, Liaocheng, Shandong, 252000, China
}

\begin{abstract}
Objective: To compare the clinical effects of minimally invasive esophageal cancer radical resection and traditional esophageal cancer radical resection. Methods: 200 cases of esophageal cancer radical resection were performed from July 2014 to July 2017 in our hospital. The cases were divided into experimental group and control group, 82 cases in the experimental group and 118 cases in the control group. The experimental group was treated with minimally invasive esophageal cancer radical surgery, and the control group was treated with conventional thoracotomy. Record the comparison between the two groups: (1) surgical conditions, including the time of surgery, intraoperative blood loss, hospitalization time; (2) the number of lymph nodes cleaned; (3) the postoperative control group used conventional thoracotomy, including lung lesions, anastomotic fistula/narrow. Results: The parameters of operation time, intraoperative blood loss, hospitalization time, and number of lymph nodes cleaned in the experimental group were lower than those in the control group, and the difference was statistically significant $(p<0.05)$. In addition to pulmonary infection $(p<0.05)$, there was no significant difference in the incidence of other complications between the experimental group and the control group $(p>0.05)$. Conclusion: Minimally invasive esophageal cancer radical resection and conventional thoracotomy have good clinical effects in the treatment of esophageal cancer. Minimally invasive esophageal cancer radical surgery can effectively reduce intraoperative trauma and postoperative reaction, which is worthy of popularization and application.
\end{abstract}

Keywords: minimally invasive esophageal cancer radical surgery; conventional thoracotomy; esophageal cancer

Publication date: November, 2018

Publication online: $30^{\text {th }}$ November, 2018

Corresponding Author: Ke Du, 511hwkj@sohu.com

\section{Introduction}

Esophageal cancer is a digestive system malignant disease, clinically very common, its global incidence is ranked eighth in malignant tumors, and its global mortality ranks sixth in malignant tumors ${ }^{[1]}$. For esophageal cancer, the current preferred treatment is surgical resection, but traditional surgical methods have long incision, limited intraoperative vision, obvious postoperative complications, severe postoperative pain, and long postoperative recovery time ${ }^{[2]}$. These defects limit the clinical application of surgical resection of esophageal cancer and cause great pain for surgical patients. In order to solve these drawbacks, more and more researchers hope to have minimally invasive surgery methods, and use laparoscopic techniques to safely and completely remove the lesions ${ }^{[3,4]}$ on the basis of reducing the wound and ensuring the surgical field of view. The purpose of this study was to compare the clinical effects of minimally invasive esophageal cancer radical resection and conventional esophageal cancer radical surgery.

\section{Data and methods}

\subsection{General information}

200 cases of esophageal cancer radical resection were performed from July 2014 to July 2017 in our 
hospital, including 112 males and 88 females with an average age of $58.6 \pm 2.3$ years. The cancer lesions were located in the upper segment in 16 cases and the middle segment in 128 cases. For example, 56 cases are located in the lower segment. The inclusion criteria were: (1) preoperative diagnosis by pathological biopsy as esophageal cancer, (2) no distant metastasis, and (3) no inoperable systemic or organic lesions. The cases were divided into experimental group and control group, 82 cases in the experimental group and 118 cases in the control group. The experimental group underwent minimally invasive esophageal cancer radical surgery, 46 males and 36 females, with an average age of $57.9 \pm 2.1$ years; 8 patients with lesions in the upper segment, 48 patients in the middle segment, and 16 patients in the lower segment; 10 patients in the i phase, 29 in the ii phase. For example, there are 43 cases in stage iii. The control group underwent routine thoracotomy. There were 66 males and 52 females with an average age of $58.9 \pm 1.9$ years. There were 12 patients with lesions in the upper segment, 75 patients in the middle segment and 25 patients in the lower segment. There were 14 patients in stage I, 40 cases in stage II, and 68 cases of III. The two groups are comparable.

\subsection{Surgical methods}

Experimental group: Inhalation combined with anesthesia, single lumen tracheal intubation. Firstly, the horizontal supine position, mark the right midline of the clavicle, set the main operation hole $2 \mathrm{~cm}$ on the umbilicus, set the auxiliary operation hole on the right rib edge, and set the auxiliary operation hole at the umbilical level of the left anterior line. With the aid of laparoscopy, the stomach is gradually freed and the lymph nodes around the stomach are swept. The cardia and esophageal hiatus are separated, part of the stomach and cardia are removed, and the remaining stomach tissue is used to make the tubular stomach. Conventional jejunostomy, drainage tube is placed in the abdominal cavity, and the abdominal cavity is closed. Subsequently, it is the left lateral position, the front line is marked, the main operation hole is placed in the fourth intercostal space, the sub operation hole is placed in the third intercostal space, and the sub operation hole is placed in the eighth intercostal space of the rear line. With the aid of thoracoscopy, the thoracic esophagus was separated and the surrounding lymph nodes were removed. Finally, the cervical esophagus is separated and anastomosed to the fundus. Place a drainage tube in the chest to close the chest. Control group: Inhalation combined with anesthesia, single lumen endotracheal intubation. The patient was in the right lateral position, and was separated into the thoracic cavity by layer 6 from the intercostal space. During the period, it was confirmed that there was no tumor invasion sign in the surrounding organs. Gradually free the esophagus and stomach, and clean the surrounding lymph nodes. Resection of the cardia and part of the stomach, parallel tubular gastroplasty. The esophagus was cut at the neck, and the tubular stomach was lifted to the neck to fit the end of the esophagus; the thoracic drainage tube was placed, and the thoracic cavity was closed layer by layer.

\subsection{Observation indicators}

Record the comparison between the two groups: (1) surgical conditions, including the time of surgery, intraoperative blood loss, hospitalization time; (2) the number of lymph nodes cleaned; (3) the postoperative control group used conventional thoracotomy, including lung lesions, anastomotic fistula/narrow.

\subsection{Statistical methods}

Data analysis was performed using SPSS 19.0 software. The quantitative data were expressed as "mean \pm standard deviation". The t-test was used for comparison between groups. The qualitative data rate (\%) was used. The chisquare test was used for comparison between groups. $\mathrm{P}<0.05$ was the difference. It is statistically significant.

\section{Results}

\subsection{Surgery}

The parameters of operation time, intraoperative blood loss and hospitalization time in the experimental group were lower than those in the control group, and the differences were statistically significant $(p<0.05)$. The specific values are shown in Table 1.

Table 1. Comparison of surgical conditions between the two groups

\begin{tabular}{cccc}
\hline Grouping & Surgical time & Intraoperative bleeding & Hospital stay \\
\hline Test group & $241.34 \pm 35.73$ & $172.47 \pm 26.41$ & $10.27 \pm 2.13$ \\
Control group & $170.36 \pm 25.68$ & $287.72 \pm 31.54$ & $16.53 \pm 2.87$ \\
t value & 16.3487 & 27.1286 & 16.7925 \\
p value & $<0.05$ & $<0.05$ & $<0.05$ \\
\hline
\end{tabular}




\subsection{Sweeping the number of lymph nodes}

The average lymph node $(15.35 \pm 2.61)$ in the experimental group and the lymph node $(10.28 \pm 1.97)$ in the control group were compared. The difference in the number of lymphatic dissection between the two groups was statistically significant $(\mathrm{t}=15.6461, \mathrm{P}<0.05)$.

\subsection{Postoperative complications}

Except for pulmonary infection $(\mathrm{p}<0.05)$, there was no significant difference in the incidence of other complications between the experimental group and the control group $(\mathrm{p}>0.05)$. The specific parameters are shown in Table 2.

Table 2. Comparison of the incidence of postoperative complications in the two groups

\begin{tabular}{cccccc}
\hline Grouping & Lung infection & Champs & Atelectasis & Anastomotic stenosis & Anastomotic fistula \\
\hline Test group & $11(13.41)$ & $2(2 . .44)$ & 0 & $10(12.20)$ & $8(9.76)$ \\
Control group & $30(25.42)$ & $4(3.39)$ & $1(0.88)$ & $8(6.78)$ & $6(5.08)$ \\
$x^{2}$ value & 4.2812 & 0.1503 & 0.6985 & 1.7325 & 1.6217 \\
p value & $<0.05$ & $>0.05$ & $>0.05$ & $>0.05$ & $>0.05$ \\
\hline
\end{tabular}

\section{Discussion}

Esophageal cancer is a common malignant tumor of the digestive tract, which is highly prevalent in Henan, Hebei and other regions ${ }^{[5]}$. Every year, hundreds of thousands of people worldwide die from esophageal cancer, and China accounts for more than half $\mathrm{f}^{[6]}$. The early symptoms of esophageal cancer are not obvious. It is usually found that the patient is already in the middle and late stage of esophageal cancer. At this time, the patient has obvious clinical symptoms, such as pain, hoarseness and weight loss during swallowing, which seriously affects the patient's survival treatment ${ }^{[7]}$.

Currently, the preferred treatment for esophageal cancer is surgical resection ${ }^{[8]}$. Because the esophagus has a special anatomy that spans the neck, chest, and abdomen, esophageal cancer cannot be considered as a common localized cancer ${ }^{[9]}$. During surgery, a large area of lymph node dissection around the operation are $\mathrm{a}^{[10]}$ should be noted to reduce cancer recurrence. The possibility of prolonging patient survival and improving the quality of life of patients. In conventional thoracotomy, the trauma is large, the postoperative recovery period is long, and the patient suffers greatly. How to reduce or even eliminate the drawbacks of traditional surgery has become an important topic of clinical concern. The clinical application of laparoscopic surgery has brought new ideas to thoracic and abdominal surgery, making it possible to make surgery more minimally invasive, clearer in vision, and more robust to operate ${ }^{[11,12]}$. The results of this study confirm this concept. The minimally invasive surgery for esophageal cancer is shorter, the intraoperative blood loss is smaller, the postoperative hospital stay is shorter, and the number of lymph nodes removed during surgery is greater $(\mathrm{p}<0.05)$. At the same time, patients in the experimental group were less likely to have pulmonary infection after surgery $(p<0.05)$. There was no significant difference in the incidence of other complications between the minimally invasive group and the conventional group $(p>0.05)$. The results show that minimally invasive esophageal cancer radical surgery is superior to conventional thoracotomy. Minimally invasive surgery requires the use of professional laparoscopic equipment, which requires the hospital to invest a large amount of money to purchase laparoscopic equipment and instruments, and the surgeon needs to receive professional skills training, which is an important factor limiting the clinical application of this procedure ${ }^{[13]}$. At the same time, due to the continuous advancement and development of laparoscopic assisted surgery technology, there is no authoritative guideline for indications and contraindications $^{[14]}$, which requires doctors to carefully select cases of minimally invasive surgery.

In summary, minimally invasive esophageal cancer radical surgery and conventional thoracotomy have a good clinical effect in the treatment of esophageal cancer. Minimally invasive esophageal cancer radical surgery can effectively reduce intraoperative trauma and postoperative response, it is worthy of popularization and application ${ }^{[15]}$.

\section{References}

[1] Wang Lidong, Hu Shoujia, Lu Shuang, et al. Progress in basic research and clinical application of esophageal cancer[J]. Henan Medical Research, 2016, 26(8):1345-1347.

[2] Shen Gang, Yue Guojun, Bai Yuju, et al. Clinical evaluation of esophageal cancer combined with radiotherapy for esophageal cancer[J]. Journal of Clinical Medicine Research 
and Practice, 2016, 1(17):60-62.

[3] Gu Xiaobo, Cong Wei. Effect of endoscopic esophageal cancer radical resection for advanced esophageal cancer[J]. Journal of Practical Hospital, 2016, 13(1):46-47.

[4] Cai Lei, Li Yan, Xiao Shu-ao, et al. Short-term efficacy evaluation of laparoscopic minimally invasive esophageal cancer for the treatment of Siewert type I gastroesophageal squamous cell carcinoma[J]. Progress in Modern Biomedicine, 2015, 15(34):6682-6685,6700.

[5] Zuo Tingting, Zheng Rongshou, Zeng Hongmei, et al. Analysis of the incidence and trend of esophageal cancer in China [J]. Chinese Journal of Oncology, 2016, 38(9):703708.

[6] Yan Bao, Zhao Jianqiang, Hou Yulong, et al. Comparative study of minimally invasive and open radical surgery for esophageal cancer in lymph node dissection and early postoperative complications [J]. Chinese Journal of Minimally Invasive Surgery, 201616(3):205-208.

[7] Li Jiandong. The effect of rapid rehabilitation surgery on minimally invasive esophageal cancer resection [J]. Health: late edition, 2015, 9 (9):121.

[8] Fu Jianhua, Tan Zihui. Current status and future prospects of surgical treatment of esophageal cancer [J]. Chinese Journal of Clinical Oncology, 2016, 43(12):507-510.

[9] Li Zhijia, Wang Junye, Zhang Haibo, et al. Effect of endoscopic esophageal cancer radical resection in the treatment of esophageal cancer $[\mathrm{J}]$. Chinese and Foreign
Medical Research, 2015, 13(3):42-43.

[10] Nie Jun, Zhou Bo, Wang Lu. Effects of different lymph node dissection on lymph node metastasis recurrence rate, survival time and complications in elderly patients with upper thoracic esophageal cancer[J]. Journal of Practical Cancer, 2017, 32(8):1267-1269.

[11] Wang Youyu, Zeng Fuchun, Cong Wei, et al. Application of thoracoscopic technique in esophageal cancer surgery [J]. Chinese Journal of Thoracic and Cardiovascular Surgery, 2015, 22(6):610-612.

[12] Fan Wenqiang. Clinical analysis of thoracic and laparoscopic combined with minimally invasive treatment of esophageal cancer[J]. Henan Medical Research, 2016, 25(2):313-314.

[13] Hou Yulong, Guo Wei, Yang Zhijian, et al. Comparative study of $3 \mathrm{~d}$ and $2 \mathrm{~d}$ thoracic laparoscopic treatment of esophageal cancer[J]. Chinese Journal of Gastrointestinal Surgery, 2015, 18(9):889-892.

[14] Wen Yuqin, He Li, Li Lixia. Prevention and control of infection in patients undergoing thoracoscopic and laparoscopic surgery combined with esophageal cancer[J]. Chinese Journal of Nosocomiology, 2016, 26(1):98-99.

[15] Liu Baoxing, Li Yin et al. Comparison of minimally invasive McKeown and left thoracic esophagectomy for the treatment of lower thoracic esophageal cancer[J]. Chinese Journal of Thoracic and Cardiovascular Surgery, 2013, 29 (6):342-345. 